\title{
aMatReader: Importing adjacency matrices via Cytoscape
}

\section{Automation [version 1; peer review: 2 approved]}

\author{
Brett Settle (D1), David Otasek1, John H Morris (D2), Barry Demchak1
}

${ }^{1}$ Department of Medicine, University of California, San Diego, California, 92093-0688, USA

2University of California San Francisco, San Francisco, California, 94143, USA

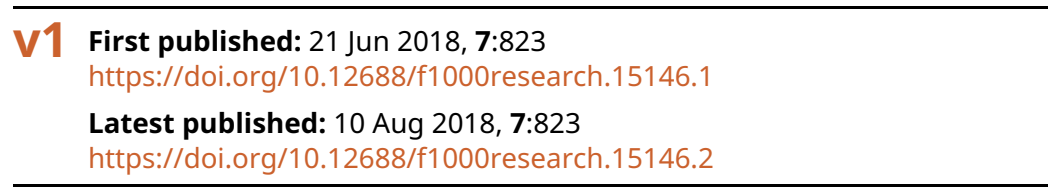

\section{Abstract}

Adjacency matrices are useful for storing pairwise interaction data, such as correlations between gene pairs in a pathway or similarities between genes and conditions. The aMatReader app enables users to import one or multiple adjacency matrix files into Cytoscape, where each file represents an edge attribute in a network. Our goal was to import the diverse adjacency matrix formats produced by existing scripts and libraries written in R, MATLAB, and Python, and facilitate importing that data into Cytoscape. To accelerate the import process, aMatReader attempts to predict matrix import parameters by analyzing the first two lines of the file. We also exposed CyREST endpoints to allow researchers to import network matrix data directly into Cytoscape from their language of choice. Many analysis tools deal with networks in the form of an adjacency matrix, and exposing the aMatReader API to automation users enables scripts to transfer those networks directly into Cytoscape with little effort.

\section{Keywords}

Workflow, Reproducibility, Cytoscape, Interoperability, REST, Microservice, Adjacency

\author{
Open Peer Review \\ Approval Status \\ 1 \\ 2 \\ version 2 \\ (revision) \\ 10 Aug 2018 \\ version 1 \\ 21 Jun 2018

$\checkmark$ \\ 1. Hideo Matsuda ID, Osaka University, Osaka, \\ Japan \\ 2. Kimberly Glass, Harvard Medical School, \\ Boston, USA \\ Any reports and responses or comments on the \\ article can be found at the end of the article.
}

This article is included in the Bioinformatics gateway.

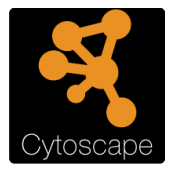

This article is included in the Cytoscape

gateway. 
Corresponding author: Barry Demchak (bdemchak@ucsd.edu)

Author roles: Settle B: Methodology, Software, Validation, Writing - Original Draft Preparation; Otasek D: Software, Validation, Writing - Review \& Editing; Morris JH: Conceptualization, Methodology, Software; Demchak B: Conceptualization, Project Administration, Software, Supervision, Writing - Review \& Editing

Competing interests: No competing interests were disclosed.

Grant information: This work was supported with funding from the National Resource for Network Biology (NRNB) under award number P41 GM103504 and the National Institute of General Medical Sciences (NIGMS) under award number R01 GM070743, both assigned to Trey Ideker.

The funders had no role in study design, data collection and analysis, decision to publish, or preparation of the manuscript.

Copyright: $\odot 2018$ Settle B et al. This is an open access article distributed under the terms of the Creative Commons Attribution License, which permits unrestricted use, distribution, and reproduction in any medium, provided the original work is properly cited.

How to cite this article: Settle B, Otasek D, Morris JH and Demchak B. aMatReader: Importing adjacency matrices via Cytoscape Automation [version 1; peer review: 2 approved] F1000Research 2018, 7:823 https://doi.org/10.12688/f1000research.15146.1

First published: 21 Jun 2018, 7:823 https://doi.org/10.12688/f1000research.15146.1 


\section{Introduction}

Adjacency matrices are a strong choice for storing pairwise element interaction data, such as those commonly produced by biological analysis tools to represent a weighted network of relationships between biological components (such as genes, conditions, pathways, times, etc.). aMatReader facilitates importing general adjacency matrices (such as correlation, similarity, and difference data) into edge attributes of Cytoscape networks. aMatReader aims to enable users to compile Cytoscape networks from one or multiple matrix files by creating edges or edge attributes for nonzero values in the matrix.

We upgraded the original aMatReader ${ }^{1}$ to enable Cytoscape Automation ${ }^{2}$ by exposing two new REST endpoints $^{3,4}$, bridging the gap between network matrix data in automation scripts and Cytoscape. With Cytoscape Automation, biologists can manipulate Cytoscape networks via REST calls and create complex workflows in their language of choice (e.g. Python and R). Researchers can then utilize Cytoscape's filtering tools to remove redundant or unremarkable edges between components, slimming the network and emphasizing stronger relationships to further their analysis.

In this paper, the Implementation section describes the general approach of aMatReader and its REST endpoints. The Operation section describes how to call the endpoint as a Cytoscape Automation Function. The Use Case section demonstrates how to import adjacency matrices into Cytoscape via the aMatReader endpoint, and the Discussion section describes the import performance.

aMatReader translates adjacency matrices into Cytoscape networks by adding edges or edge attributes represented by non-null values in the matrix. The square adjacency matrix is the standard matrix representation of a network. In a square matrix, node labels are stored in the first row and column of a table of size $(\mathrm{N}+1, \mathrm{~N}+1)$. The $N \times N$ grid of values within the table contains edge weights. A non-null value at cell $(i, j)$ represents the weight of an edge between node $i$ and node $j$. An example matrix text file and graph representation can be seen in Figure 1.

\begin{tabular}{|l|l|l|l|l|}
\hline Rows & A & B & C & D \\
\hline A & 0 & 2 & 0 & 3 \\
\hline B & 2 & 0 & 1 & 2 \\
\hline C & 0 & 1 & 0 & 0 \\
\hline D & 3 & 2 & 0 & 0 \\
\hline
\end{tabular}

a) Square adjacency matrix as a grid

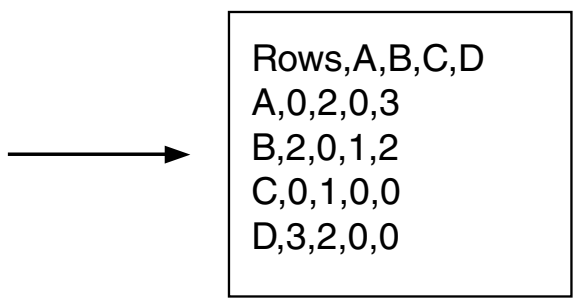

b) Matrix written to text file

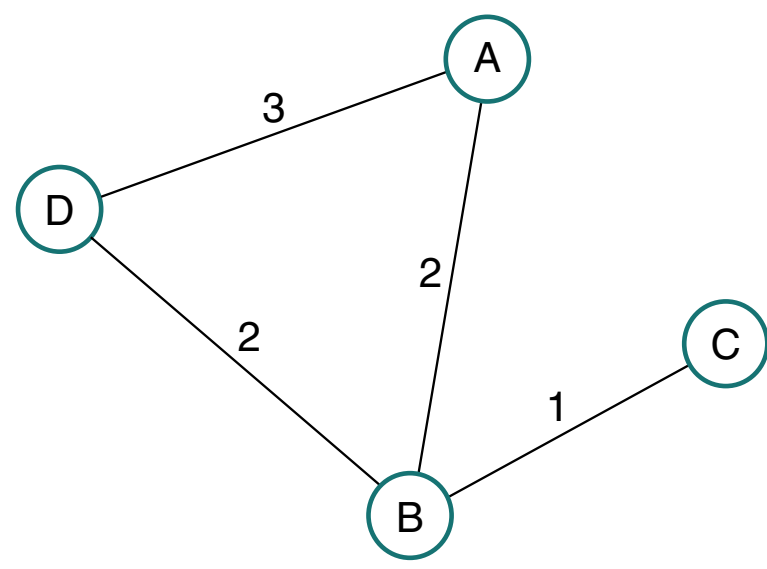

c) Imported to Cytoscape Network

Figure 1. Converting a square adjacency matrix into an undirected network. (a) In Excel, Python, etc., the matrix is stored as a 2-dimensional array with optional labels. (b) The matrix is exported to a comma delimited file. (c) Importing the undirected matrix into Cytoscape with aMatReader, edges are defined by nonzero values within the upper triangle. 
If an analysis tool is calculating distance or similarity between all pairs of genes within pathways, it will likely produce a square adjacency matrix. Some other examples of square matrices are covariance and correlation matrices. Square matrices are often symmetric, meaning row and column names are similarly ordered, the value at cell $(i, j)$ is the same as that at $(j, i)$, and the values along the diagonal are calculated by comparing an element to itself. A symmetric matrix represents an undirected network, and only refers to the upper triangle for edge attributes.

In the case of diagonal square matrices, it can be efficient to omit row names, because the row name at index $i$ is identical to the $i^{\text {th }}$ column name. This is especially useful when exporting matrices from Python using numpy by inserting node names to the file as a header row. (ref Listing 1 numpy example)

However, there are many cases where an adjacency matrix is not square. For example, a correlation matrix between genes and conditions will have different row elements than column elements. The network generated by such a correlation matrix will be directed and bipartite. As a directed network, the entire matrix is used to generate edge attributes, unlike undirected networks that only use the upper triangle.

\section{Methods}

Implementation

aMatReader is developed to handle a wide spectrum of adjacency matrix formats. To accommodate possible missing or reordered row and column names, we use an adjacency list of node indices as an intermediary data structure. Two separate arrays are used to store source and target node names, where the $i^{\text {th }}$ name in the array refers to the node at index $i$ in the adjacency list. Once the matrix has been translated, aMatReader makes a pass through the adjacency list and set edge attributes in the network.

One constraint of aMatReader is that the parser expects integer or floating point values. Any String, Boolean, or unrecognized values will be considered null and no edge will be created (and no warning will be generated).

There are two possible options for importing an adjacency matrix into a Cytoscape network. To create a new network from an adjacency matrix file, the caller can use the import Function. If the adjacency matrix defines edge attributes that should be added to an existing network, the extend Function should be used. This is especially useful because an adjacency matrix can only represent one type of edge attribute, and complex networks are often represented by multiple adjacency matrix files.

If a new network is being created (via the import Function, described in the Operation section below), all of the network nodes are created and named first. Then each non-null value in the matrix is used to create an edge. The edge attribute takes its name from the name of the matrix file that is being imported.

If a network is being extended (via the extend Function, described in the Operation section below), aMatReader attempts to match row and column names to existing nodes in the network. If no node exists with the given name, a new one is created. Creating edge attributes is handled similarly; an edge between the source and target node is added if it does not already exist, and then the attribute is set.

Some matrix formats add extra information to provide insight to the parser. Matrices produced by cCrepe and MATLAB optionally prefix column names with a period-delimited description of the weights specified by the matrix (e.g. "sim.score" or "q.value" in cCrepe). Additionally, comments can be included in files by inserting a hash symbol at the start of a line. Below is an example gene similarity table produced by MATLAB. More examples of supported format idiosyncrasies can be seen in the documentation provided on the Cytoscape App Store.

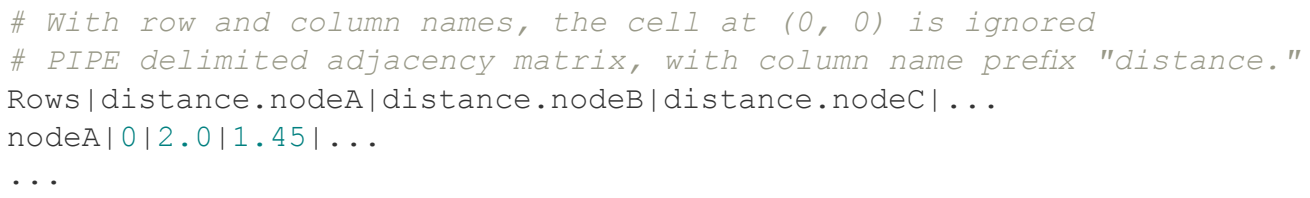

Listing 1. Sample adjacency matrix with confusing format. Pipe "|" delimited text file with comments and column name prefix.

Operation

aMatReader exposes two Functions ${ }^{5}$ via the Cytoscape CyREST API, import to create a new network and extend to add edge attributes to an existing network. If necessary, the caller specifies the network to be extended 
as part of the REST URL. The Function endpoints (Figure 2) enable users to manipulate network data as internal Cytoscape data, and are documented in the Apps: aMatReader section of the Swagger document available via Help $\rightarrow$ Automation $\rightarrow$ CyREST API.

Both endpoints expect the same parameters within the JSON body of the request:

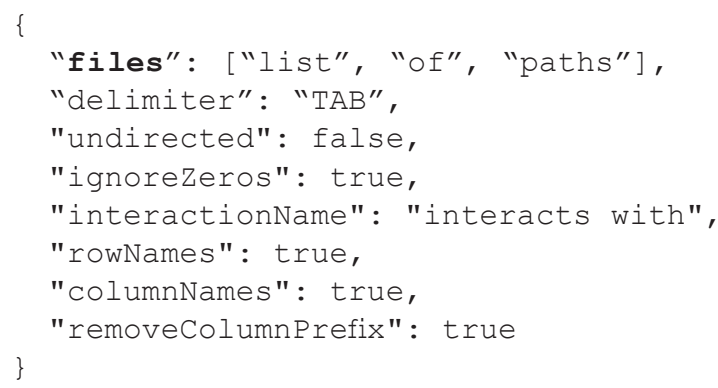

The files parameter specifies a list of local file paths for matrix files, and is the only required parameter; all other parameters default to the values shown above. Files imported in the same REST call must have the same format and thus be importable with identical parameters. The caller can specify the matrix delimiter (as one of "PIPE", "SPACE", "TAB", or "COMMA"), whether the matrix is symmetric and diagonal and should only import the upper triangle as undirected, whether or not to create edges for zero values (called ignoreZeros), edge interaction type (called interactionName). The payload can also define whether row and column names are present in the file (called rowNames and columnNames). The removeColumnPrefix parameter informs the parser to ignore a common prefix in column names, if it exists.

Note that the interaction type is only set for edges created by the import, and is not set for pre-existing edges in an extend call.

The aMatReader endpoints return a CIResponse ${ }^{6}$ according to Cytoscape Automation best practices. If the call succeeds, the CIResponse contains an import result object (as the data element); otherwise, it contains an explanation of the error (as the errors element):

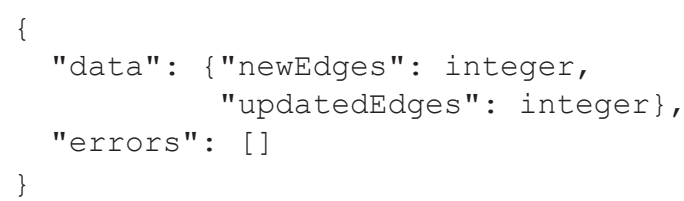

The newEdges value contains the number of edges created in the network, and the updatedEdges contains the number of edges that already existed and received new edge attribute(s).

If the delimiter is unrecognized or any of the matrix files cannot be found or fails to parse correctly, the errors[0].status element returns 404, and the remainder of the errors[0] element contains additional information.

In order to download and use aMatReader, ensure that you are running Cytoscape version 3.6.0 or later with at least $512 \mathrm{MB}$ of free memory to store the matrix before creating the edges.

Calling aMatReader Functions. To import files to a new network, the caller must send an HTTP POST request to /v1/aMatReader/import with a JSON payload object specifying the list of matrix files and any optional 
parameters listed above. To extend an existing network, the caller must also pass the networkSUID parameter as part of the URL (/v1/aMatReader/extend/\{networkSUID\}).

Note that the networkSUID must be an integer. The caller can determine a network's SUID via the /v1/networks endpoint.

Example code is provided in R, Python and as a Bash curl, but can easily be adapted into any language that supports REST calls.

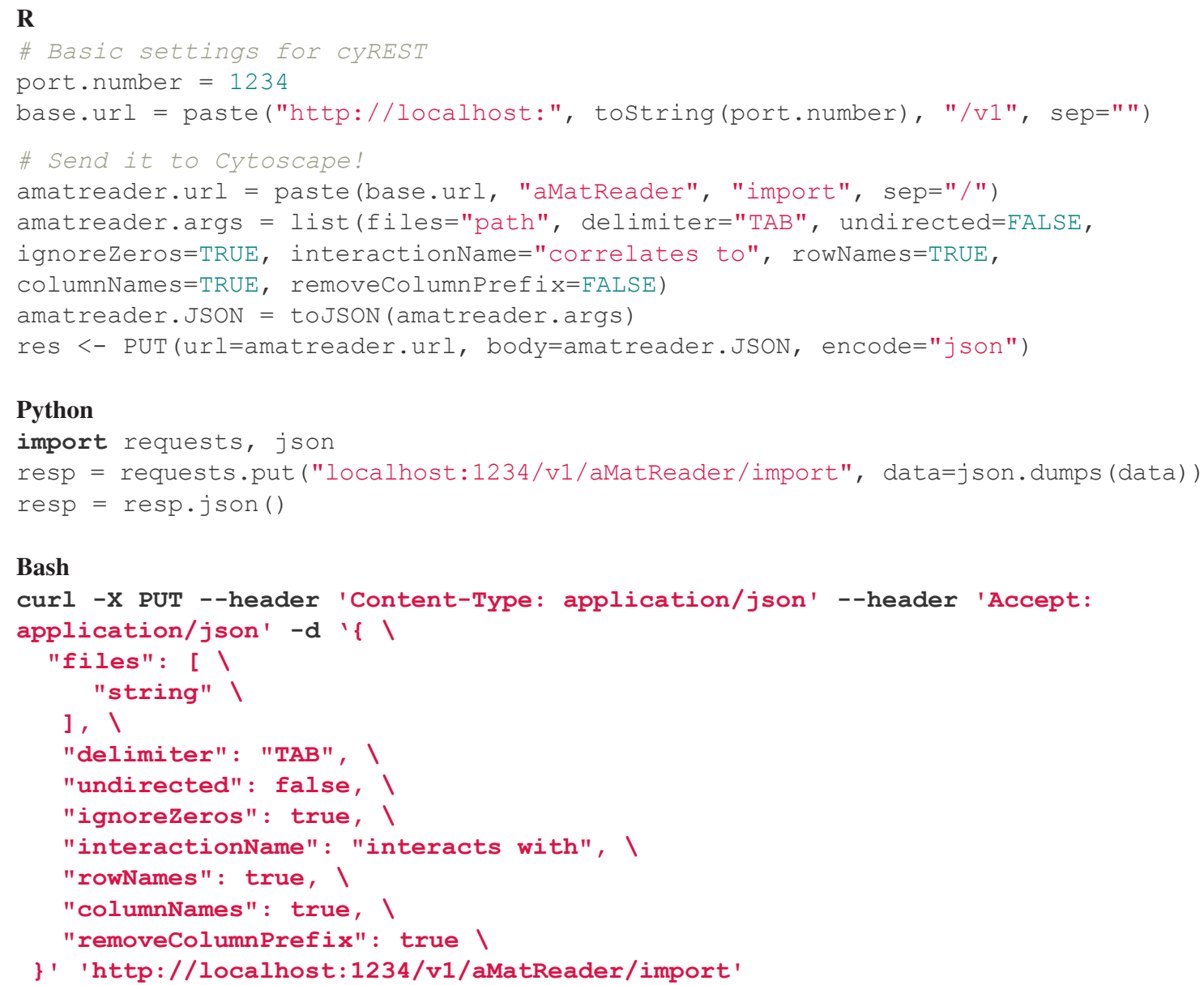

\section{Bash}

curl -X PUT --header 'Content-Type: application/json' --header 'Accept:

\section{Use cases}

The simple use case that inspired an upgrade to the original aMatReader app was filtering correlation data in search of similarities among different stages of disease severity. The $\mathrm{R}$ package cCrepe gives compositionally corrected scores for all pairwise connections in a dataset, producing an adjacency matrix for similarity score and q-values. Both files contained row names, as well as column names prefixed by "sim.score" or "q.value", respectively. aMatReader allows the user to import both files into one Cytoscape network, which can easily be filtered with a few extra calls to the core Cytoscape CyREST API (as shown below):

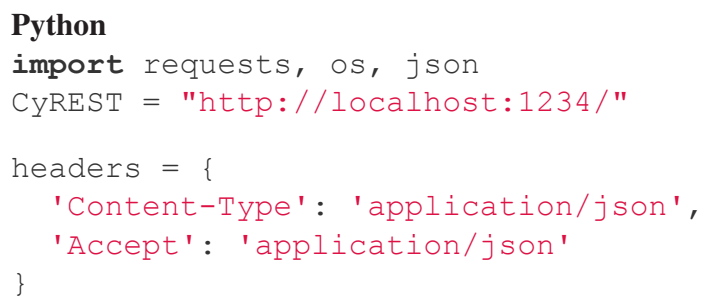




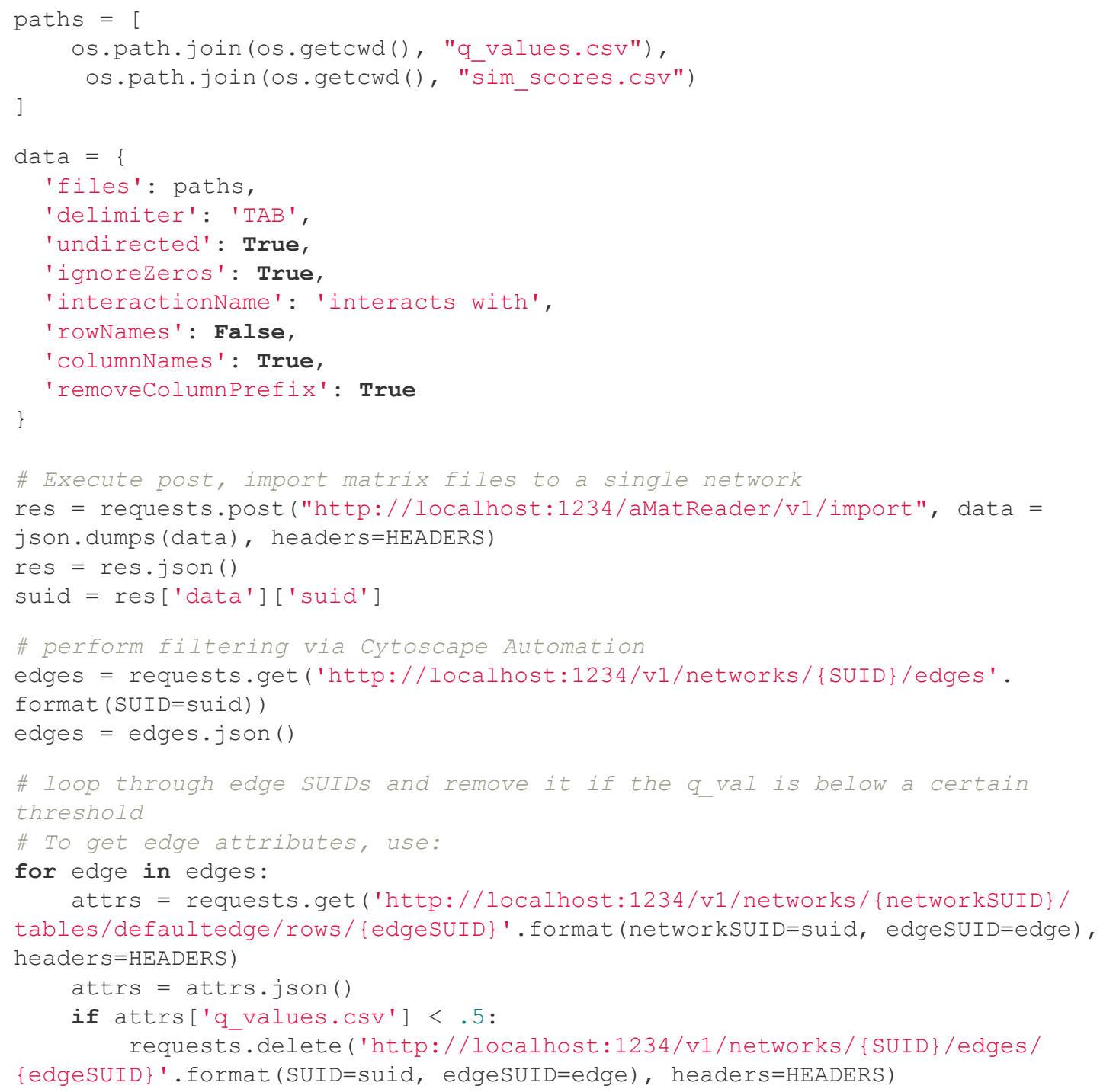

The response to the aMatReader function call will create a Cytoscape network with edges that each have a sim_score and q_val column. With a few extra lines, the script can perform filtering and analysis without any interaction from the user. Exposing the aMatReader API enables the researcher to completely automate their analysis process without leaving their script.

\section{Discussion}

Translating from matrix to adjacency list representation decreases the space from $\mathrm{O}\left(\mathrm{N}^{\wedge} 2\right)$ to $\mathrm{O}(\mathrm{N}+\mathrm{E})$ for a network with $\mathrm{N}$ nodes and $\mathrm{E}$ edges, and the time complexity is similarly decreased for adding the edge attributes to the network.

\section{Future plans}

aMatReader was designed with existing adjacency matrix exporters in mind, such as the igraph.Graph.Adjacency ${ }^{7}$ and pandas DataFrame ${ }^{8}$ objects. We will continue to improve aMatReader to handle more diverse matrix formats, including string matrices, and to provide a CyREST Function that returns the predicted parameters for importing a matrix file.

\section{Summary}

In this paper, we present aMatReader, a general adjacency matrix importer app for Cytoscape 3. aMatReader creates another data entry method that allows researchers to join multiple adjacency matrices, each representing an edge attribute, into one network. aMatReader was developed to handle importing diverse matrix file formats into Cytoscape via automation scripts and libraries. 
The aMatReader CyREST API is composed of two import Functions that enable users to create a new network or extend an existing network with an adjacency matrix defining edge attributes. Users can also import multiple adjacency matrices that represent different edge attributes in the same network simultaneously before calling Cytoscape's advanced filtering functions.

\section{Data availability}

All data underlying the results are available as part of the article and no additional source data are required.

\section{Software availability}

The aMatReader app is available on the Cytoscape App Store: http://apps.cytoscape.org/apps/aMatReader.

Source code available from: https://github.com/idekerlab/aMatReader.

Archived source code available from: https://doi.org/10.5281/zenodo.1287303

License: GNU Lesser General Public License v2.1.

Competing interests

No competing interests were disclosed.

Grant information

This work was supported with funding from the National Resource for Network Biology (NRNB) under award number P41 GM103504 and the National Institute of General Medical Sciences (NIGMS) under award number R01 GM070743, both assigned to Trey Ideker.

The funders had no role in study design, data collection and analysis, decision to publish, or preparation of the manuscript.

\section{Acknowledgments}

We would like to thank Annika Kroeger for bringing more adjacency matrix use cases to our attention and inspiring the updates to aMatReader.

1. Cytoscape App Store - aMatReader. [cited 22 May 2018]. Reference Source

2. cytoscape-automation. Github. Reference Source

3. Fielding RT, Taylor RN: Principled Design of the Modern Web Architecture. ACM Trans Internet Technol. New York, NY, USA: ACM; 2002; 2: 115-150. Publisher Full Text

4. Ono K, Muetze T, Kolishovski G, et al: CyREST: Turbocharging Cytoscape Access for External Tools via a RESTful API [version 1; referees: 2 approved]. F1000Res. 2015; 4: 478 PubMed Abstract | Publisher Full Text | Free Full Text

5. Cytoscape Automation FAQ - What is the difference between Commands and Functions? In: Google Docs. [cited $18 \mathrm{Apr}$
2018]

Reference Source

6. App Developers: Cytoscape Function Best Practices - Cl Response. [cited 18 Apr 2018]. Reference Source

7. Csardi G, Nepusz T: The igraph software package for complex network research. Inter Journal, Complex Systems. 2006; 1695: 1-9. Reference Source

8. McKinney W: Python for Data Analysis: Data Wrangling with Pandas, NumPy, and IPython. "O'Reilly Media, Inc."; 2012. Reference Source

9. Settle B, Morris S: idekerlab/aMatReader: aMatReader v1.1.3 (Version v1.1.3). Zenodo. 2018.

Data Source 


\section{Open Peer Review}

\section{Current Peer Review Status:}

\section{Version 1}

Reviewer Report 03 July 2018

\section{https://doi.org/10.5256/f1000research.16500.r35307}

(C) 2018 Glass K. This is an open access peer review report distributed under the terms of the Creative Commons Attribution License, which permits unrestricted use, distribution, and reproduction in any medium, provided the original work is properly cited.

\section{Kimberly Glass}

Department of Medicine, Harvard Medical School, Boston, MA, USA

this is a clearly-written summary of the updated aMatReader API. This tool will be useful for reading in certain types of networks (e.g. correlation networks) into Cytoscape.

A few additional thoughts:

A description of how the tool works from within Cytoscape would be a nice addition to the paper for the less-computationally-savvy users of Cytoscape.

It would be useful for the authors to clarify that for directed networks, rows in the input file represent "source" nodes and columns "target" nodes.

I found the discussion section too short. Additional discussion on how aMatReader overcomes the limitation described, and/or when there is an advantage to the matrix format over an adjacency list representation would be useful.

Is the rationale for developing the new software tool clearly explained? Yes

Is the description of the software tool technically sound?

Yes

Are sufficient details of the code, methods and analysis (if applicable) provided to allow replication of the software development and its use by others?

Yes

Is sufficient information provided to allow interpretation of the expected output datasets and any results generated using the tool?

Yes 
Are the conclusions about the tool and its performance adequately supported by the findings presented in the article?

Yes

Competing Interests: No competing interests were disclosed.

\section{I confirm that I have read this submission and believe that I have an appropriate level of expertise to confirm that it is of an acceptable scientific standard.}

Author Response 02 Aug 2018

\section{Brett Settle}

We would like to thank the referee for the positive feedback and respond to the very helpful comments:

We wrote this publication as part of a collection of Cytoscape Automation papers, which focus on enabling researchers to programmatically access CyREST endpoints exposed by apps, over Cytoscape Desktop access. However, I agree that it would be useful to include a section for non-programmers near the beginning.

Thank you for noticing this omission. We will add the row/column information to the paper.

We will be sure to add more information to the discussion section in the near future. The current implementation of aMatReader translates the matrix to a mapping to better handle sparse matrices. Cytoscape core network importer supports adjacency lists so that format would be preferred, but some workflows output one or many adjacency matrices that need to be imported into Cytoscape all at once.

Competing Interests: No competing interests were disclosed.

Reviewer Report 02 July 2018

https://doi.org/10.5256/f1000research.16500.r35308

(C) 2018 Matsuda H. This is an open access peer review report distributed under the terms of the Creative Commons Attribution License, which permits unrestricted use, distribution, and reproduction in any medium, provided the original work is properly cited.

\section{Hideo Matsuda}

Graduate School of Information Science and Technology, Osaka University, Osaka, Japan

This paper presents a tool for importing adjacency matrices into Cytoscape. The tool is particularly useful to represent weighted biological networks, such as co-expression relationships of genes, confidence scores of predicted relationships, etc.

Minor comment: In "files" parameter in the JSON code, the character codes of some double quotes 
(") are different from others.

Is the rationale for developing the new software tool clearly explained?

Yes

Is the description of the software tool technically sound?

Yes

Are sufficient details of the code, methods and analysis (if applicable) provided to allow replication of the software development and its use by others?

Yes

Is sufficient information provided to allow interpretation of the expected output datasets and any results generated using the tool?

Yes

Are the conclusions about the tool and its performance adequately supported by the findings presented in the article?

Yes

Competing Interests: No competing interests were disclosed.

Reviewer Expertise: gene expression analysis, gene regulatory network inference

I confirm that I have read this submission and believe that I have an appropriate level of expertise to confirm that it is of an acceptable scientific standard.

\section{Author Response 02 Aug 2018}

\section{Brett Settle}

We would like to thank the referee for positive feedback and helpful comments. The inconsistent character codes will be fixed.

Competing Interests: No competing interests were disclosed. 
The benefits of publishing with F1000Research:

- Your article is published within days, with no editorial bias

- You can publish traditional articles, null/negative results, case reports, data notes and more

- The peer review process is transparent and collaborative

- Your article is indexed in PubMed after passing peer review

- Dedicated customer support at every stage

For pre-submission enquiries, contact research@f1000.com 